\title{
Evaluation of the degradation of fine asphalt-aggregate mixtures containing high Reclaimed Asphalt Pavement (RAP) contents
}

Diana B. Sánchez*a, James Grenfell ${ }^{\mathrm{a}}$, Gordon Airey and Silvia Caro ${ }^{\mathrm{b}}$

${ }^{a}$ Nottingham Transportation Engineering Centre (NTEC), Pavement Research Building, University of Nottingham, University Park, NG7 2RD, Nottingham, UK

${ }^{b}$ Department of Civil and Environmental Engineering, Universidad de los Andes, Carrera 1 Este \#19A-40, Edificio ML, Bogotá D.C., Colombia

E-mail address*: evxdbs@nottingham.ac.uk 


\title{
Evaluation of the degradation of fine asphalt-aggregate mixtures containing high Reclaimed Asphalt Pavement (RAP) contents
}

\author{
This paper evaluates the mechanical properties and performance of the Fine \\ Aggregate Matrix (FAM) existing within full RAP asphalt mixtures, in terms of \\ their rheological and fatigue deterioration properties. The RAP material was \\ produced in the laboratory to control its properties and to reduce the effects of \\ variability associated with these materials. Four FAM mixtures were analysed, \\ including a virgin HMA, a 100\% RAP and two mixtures containing 50\% RAP in \\ combination with virgin materials using different penetration grade virgin binders. \\ The analysis of the deterioration properties was based on the application of a \\ fracture model that incorporates the viscoelastic properties of the material, the \\ quality of the adhesive bonds developed between the aggregates and the \\ corresponding bitumen present in each mixture, and the rate at which the material \\ dissipates energy when subjected to cycling loading. The input parameters for this \\ model include the results obtained from Dynamic Mechanical Analysis (DMA) \\ and Surface Free Energy (SFE) tests. In general, the results showed that the \\ incorporation of 50\%RAP content increased the stiffness of the final mixtures, as \\ expected. However, this hardening effect did not result in mixtures with inferior \\ fatigue performance of the FAM present in the full mixtures, at the applied strain \\ level.
}

Keywords: Reclaimed Asphalt Pavement (RAP); Fine Aggregate Matrix (FAM); Dynamic Mechanical Analysis (DMA), Surface Free Energy (SFE), Viscoelastic properties, Fatigue.

\section{Introduction}

Reclaimed Asphalt Pavement (RAP) materials are essentially flexible pavements that have reached the end of their service lives and are reclaimed to be used as part of new or rehabilitated road structures. The incorporation of this material in Hot Mix Asphalt (HMA) mixtures can reduce the amount of new material that has to be added to the final mixture, generating possible cost savings and contributing to the conservation of natural resources (Copeland, 2011). Undoubtedly, these factors make this practice a valuable 
alternative for the construction of road infrastructure projects. Typical values of RAP allowed in the fabrication of new asphalt mixtures are in a range of 10 to $20 \%$ (Mogawer et al., 2012). However, concerns related to the contribution of the hardened bitumen from RAP in the final mixture performance and durability in the field have limited the production of new HMA with higher amounts of this material.

One of the reasons for this limitation is related to the unclear role that the aged bitumen from RAP plays in the presence of virgin materials. The aging process of the bitumen during its life service causes an increase in its viscosity, and subsequent stiffening of the asphalt mixture (Bell, 1989). As a result, there is a common belief that the use of this material can lead to poor field performance especially because the increased brittleness could negatively affect its fatigue and thermal cracking resistance. This belief has been supported by some authors (Bonaquist, 2005; Daniel, Pochily, \& Boisvert, 2010; McDaniel, Shah, Huber, \& Gallivan, 2007), and refuted by others (Hajj, Sebaaly, \& Shrestha, 2007; Maupin, Diefenderfer, \& Gillespie, 2008; Tabakovi, Gibney, Mcnally, \& Gilchrist, 2010). Moreover, another concern related with the use of RAP materials is associated with the interaction between the aged bitumen present in the RAP and the virgin bitumen. A number of studies related to this concern have been conducted (Copeland, 2011; Huang, Li, Vukosavljevic, Shu, \& Egan, 2005; Mogawer et al., 2012), which have mainly focused on determining the level of blending between old-new bitumen and how this process, along with the amount of RAP introduced, affects the performance of the mixture. However, there are other issues related with this interaction of old-new materials that still remain unsolved and play a significant role in the performance of the final mixtures. Particularly, these issues are related to the adhesion characteristics that the old, hardened bitumen from RAP develops with the virgin bitumen and with the new aggregates. 
Having a comprehensive characterisation of these materials and understanding the contribution of each of the components in the final mixture performance as well as the interaction between them contributes to the responsible use of this practice. These issues have become particularly important taking into account that many countries have increased the amount of RAP percentage, in what is known as high RAP content (i.e. more than 20\%) (e.g. Daniel et al. 2010; Mogawer et al. 2012; Anderson \& Daniel 2013; Bennert et al. 2014).

The aim of this paper is to conduct a comprehensive characterisation of RAP mixtures manufactured with high-RAP content (i.e. 50\%) and two penetration grade virgin binders. An unmodified control HMA and a 100\% RAP mixture were also evaluated for comparison. The effect of high-RAP content on the RAP mixtures was evaluated by means of three aspects: 1) the linear viscoelastic properties in the frequency domain by means of master curves, 2) the fatigue degradation properties of the mixtures by means of a non-linear viscoelastic fracture model that combines their fundamental viscoelastic properties, the velocity of energy dissipation during the degradation process, and the adhesive bond properties of the components of the mixtures (i.e. aggregates with virgin bitumen, with bitumen from RAP or with the final blend between the old-new bitumen in the 50\%RAP mixtures), and, 3) the fatigue resistance of the material.

The study was not conducted directly on the HMA or RAP mixtures but on the fine aggregate matrix (FAM) or asphalt mortar existing within the full mixtures. FAM is usually defined as the combination of bitumen with the finest portion of the aggregates (e.g. particles size smaller than $1.18 \mathrm{~mm}$ ) which are found in the entire asphalt mixture and have a relatively more uniform internal structure compared to the HMA (Caro, Beltrán, Alvarez, \& Estakhri, 2012; Caro, Masad, Airey, Bhasin, \& Little, 2008; Masad, Castelo Branco, Little, \& Lytton, 2008). There is only one study on RAP mixtures that 
has been conducted by testing these FAM materials (He, Alavi, Jones, \& Harvey, 2016); however, the study of this material is particular relevant since it is considered that most damage processes in HMA, especially those related to fatigue cracking and moisture damage, initiates and propagates through this fine matrix (Montepara, Romeo, Isola, \& Tebaldi, 2011).

In the following section the materials and design of the FAM mixtures including RAP are presented. Then, the rheological properties of these mixtures are presented in the form of master curves. Next, the fracture model used to evaluate their fatigue resistance is presented, followed by the experimental tests performed to obtain the model parameters. Finally, the main findings and conclusions of this work are presented.

\section{Materials and design of FAM mixtures}

The FAM mixtures were designed following the methodology proposed by Masad, Zollinger, Bulut, Little, \& Lytton (2006). They simulate the fine portion of the original full HMA mixture, a 0/14 mm size close graded surface course based on the British Standard BS 4987-1:2005 containing 5\% by weight of bitumen. The bitumen content for all the FAM mixtures was approximately $10.7 \%$ by weight of the total FAM mixture, and they contain fine limestone aggregates (between $0.125 \mathrm{~mm}$ and $1 \mathrm{~mm}$ ) and limestone filler (less than 65 microns) in the proportions specified in Table 1.

Table 1. Characteristics of the FAM mixtures

\begin{tabular}{lcc}
\hline & Mass (\%) & Mass $(\mathrm{g})$ \\
Mass retained on sieve $0.5 \mathrm{~mm}(\mathrm{~g})$ & 47 & 1372 \\
Mass retained on sieve $0.25 \mathrm{~mm}(\mathrm{~g})$ & 23.5 & 686 \\
Mass retained on sieve $0.125 \mathrm{~mm}(\mathrm{~g})$ & 11.7 & 342 \\
Mass retained on sieve $0.063 \mathrm{~mm}(\mathrm{~g})$ & 5.8 & 169 \\
Filler (g) & 12 & 350 \\
Total aggregate batch & 100 & 2919 \\
Bitumen (g) & 10.7 & 350 \\
Maximum specific gravity, Gmm & $2284 \mathrm{~kg} / \mathrm{m}^{3}$ \\
Bulk specific gravity, Gmb & $2056 \mathrm{~kg} / \mathrm{m}^{3}$ \\
Air void content & \multicolumn{2}{c}{$10 \%$} \\
\hline
\end{tabular}


Four FAM mixtures were manufactured in the laboratory with this aggregate composition and bitumen content. These mixtures include a virgin HMA mixture - no RAP material -, a 100\% RAP mixture, and two mixtures composed of 50\% RAP and $50 \%$ virgin materials using two virgin binders with different penetration grades, in order to obtain mixtures with a certain penetration grade for the final bitumen blend. All mixtures were mixed at $160^{\circ} \mathrm{C}$. The control HMA mixture was fabricated using a $70 / 100$ penetration grade bitumen. In order to characterise this bitumen, penetration at $25^{\circ} \mathrm{C}(\mathrm{BS}$ 2000-49:2007), softening point (BS 2000-58:2007), rotational viscosity at $150^{\circ} \mathrm{C}$ (BS 2000-505:2010) and Fraass breaking point (BS 2000-80:2007) tests were performed. The same tests were performed on the recovered bitumen from the RAP material. The results from both binders are presented in Table 2.

Table 2. Properties of the 70/100 pen virgin bitumen and recovered bitumen from RAP

\begin{tabular}{lcccc}
\hline Bitumen & $\begin{array}{c}\text { Penetration at } \\
25^{\circ} \mathrm{C} \\
{[\mathrm{dmm}]}\end{array}$ & $\begin{array}{c}\text { Softening } \\
\text { point } \\
{\left[{ }^{\circ} \mathrm{C}\right]}\end{array}$ & $\begin{array}{c}\text { Rotational } \\
\text { Viscosity @ } 150^{\circ} \mathrm{C} \\
{[\mathrm{mPa} \cdot \mathrm{s}]}\end{array}$ & $\begin{array}{c}\text { Fraass breaking } \\
\text { point } \\
{\left[{ }^{\circ} \mathrm{C}\right]}\end{array}$ \\
\hline $\begin{array}{l}\text { Virgin bitumen } \\
\begin{array}{l}\text { Recovered } \\
\text { bitumen }\end{array}\end{array}$ & 73 & 46 & 140 & -12 \\
\hline
\end{tabular}

The RAP material used in this study was produced in the laboratory with the aim of controlling the characteristics of its components (i.e. RAP aggregate gradation, bitumen grade, etc.), while reducing issues related with the variability of their properties that could affect the reliability of the results. The laboratory procedure for FAM-RAP production consisted of subjecting a loose sample of FAM mixture to a controlled artificial long-term ageing protocol in the laboratory. Initially, the FAM mixture was manufactured with the same composition (i.e. bitumen content and aggregate gradation) as the virgin HMA presented in Table 1 at the same mixing temperature (i.e. $160^{\circ} \mathrm{C}$ ). Then, the FAM loose mixture was subjected to an ageing protocol which consisted on exposing the FAM loose mixture to heat in the oven for 32 hours (non-continuous ageing: 
$8 \mathrm{~h}$ periods during 4 days) at $105^{\circ} \mathrm{C}$, as suggested by Oke (2011). Once the RAP material was produced, the bitumen was recovered by distillation following the BS EN 126974:2005, and characterised by performing the same tests as for the virgin bitumen (Table 2).

\subsection{Bitumen blend design and FAM mixtures}

According to the European Standard for reclaimed asphalt EN 13108-8:2005, when using more than $10 \%$ of RAP for surface courses and more than $20 \%$ for binder courses in combination with virgin bitumen (i.e. unmodified), the penetration in the resulting bitumen blend could be determined through the following equations:

$$
A \log \text { pen }_{1}+B \log \text { pen }_{2}=(A+B) \log \text { pen }_{\text {blend }}
$$

where pen $_{1}$ is the penetration of the recovered bitumen from RAP; pen 2 is the penetration of the virgin bitumen, pen blend is the penetration of the final bitumen blend in the mixture containing RAP, and $A$ and $B$ are the ratios by mass of the bitumen from the RAP and of the virgin bitumen respectively $(A+B=1)$. Similarly, the softening point of the virgin and RAP blended bitumen can be computed as:

$$
T_{R \& B_{\text {blend }}}=A T_{R \& B_{1}}+B T_{R \& B_{2}}
$$

where $T_{R \& B_{b l e n d}}$ is the softening point in the final bitumen blend, $T_{R \& B_{1}}$ is the softening point of the recovered bitumen from RAP and $T_{R \& B_{2}}$ is the softening point of the virgin bitumen, $A$ and $B$ represent the same ratios as in equation (1).

For the mixtures containing 50\% RAP, equations (1) and (2) were used to determine the properties of the expected final bitumen blend using two approaches: 1) predicting the required properties of the virgin bitumen to obtain a mixture of 70/100 pen (i.e. equal to the virgin bitumen in the HMA mixture for comparison), labelled as $50 \% \mathrm{RAP}_{1}$ and 2 ) determining the properties of the final bitumen blend resulting from 
blending RAP material with the same virgin bitumen used for the control HMA mixture (i.e. the blend between the original 70/100 pen and the bitumen recovered from RAP), labelled as $50 \% \mathrm{RAP}_{2}$. Table 3 shows the properties of each component of the final bitumen blend using both approaches.

Table 3. Bitumen blend design for FAM mixtures with 50\% RAP content

\begin{tabular}{lcccc}
\hline \multirow{2}{*}{ Bitumen } & \multicolumn{2}{c}{ Penetration at $25^{\circ} \mathrm{C}(\mathrm{dmm})$} & \multicolumn{2}{c}{ Softening point $\left({ }^{\circ} \mathrm{C}\right)$} \\
\hline & $50 \% \mathrm{RAP}_{1}$ & $50 \% \mathrm{RAP}_{2}$ & $50 \% \mathrm{RAP}_{1}$ & $50 \% \mathrm{RAP}_{2}$ \\
\cline { 2 - 5 } Virgin bitumen & 141 & 73 & 39 & 46 \\
Recovered bitumen & 38 & 38 & 53 & 53 \\
Estimated final bitumen & 73 & 52 & 46 & 49 \\
\hline
\end{tabular}

\subsection{Characteristics of the FAM mixtures}

Based on the information presented previously, Table 4 summarizes the characteristics of the four FAM mixtures evaluated in this study. All mixtures were mixed and compacted at 160 and $140^{\circ} \mathrm{C}$ respectively. The amount of RAP material required for the 50\%RAP mixtures was heated up to $160^{\circ} \mathrm{C}$ for 6 hours prior its incorporation with the virgin material.

Table 4. Characteristics of the FAM and FAM-RAP mixtures

\begin{tabular}{|c|c|c|c|c|c|}
\hline \multirow[b]{2}{*}{ Mixture } & \multicolumn{3}{|c|}{ 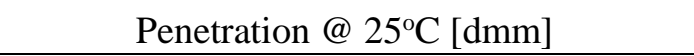 } & \multirow[b]{2}{*}{ Aggregates } & \multirow{2}{*}{$\begin{array}{c}\text { RAP } \\
\text { content } \\
{[\%]}\end{array}$} \\
\hline & $\begin{array}{l}\text { Virgin } \\
\text { bitumen }\end{array}$ & $\begin{array}{c}\text { RAP } \\
\text { bitumen }\end{array}$ & $\begin{array}{l}\text { Estimated final } \\
\text { bitumen blend }\end{array}$ & & \\
\hline Virgin HMA & 73 & - & 73 & Limestone & 0 \\
\hline $50 \% \mathrm{RAP}_{1}$ & 141 & 38 & 73 & Limestone & 50 \\
\hline $50 \% \mathrm{RAP}_{2}$ & 73 & 38 & 52 & Limestone & 50 \\
\hline $100 \%$ RAP & - & 38 & 38 & Limestone & 100 \\
\hline
\end{tabular}

\section{Linear viscoelastic properties of the FAM-RAP mixtures}

\subsection{Experimental set-up and sample preparation}

Cylindrical specimens for each of the four FAM mixtures were prepared following the method originally proposed by Masad et al. (2006). In this method, a cylindrical FAM specimen of $150 \mathrm{~mm}$ in diameter and $90 \mathrm{~mm}$ in height is fabricated using the Superpave 
gyratory compactor (SGC). After compaction, the top and bottom parts of this specimen are trimmed off, and a coring barrel is used to extract cylindrical samples of $50 \mathrm{~mm}$ in height and $12.5 \mathrm{~mm}$ in diameter. In this case, the SGC specimens were compacted following the BS EN 12697-31:2007, targeting a density of $2056 \mathrm{~kg} / \mathrm{m}^{3}$ (i.e. a target air void content of the FAM mixtures of 10\%). Figure 1 illustrates the procedure for specimens' preparation.

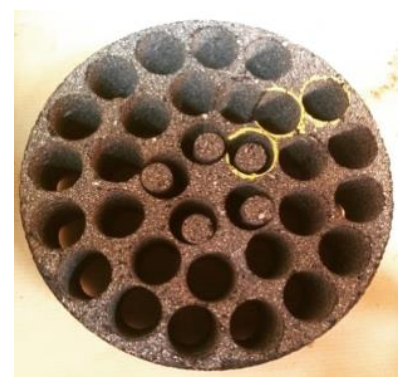

(a)

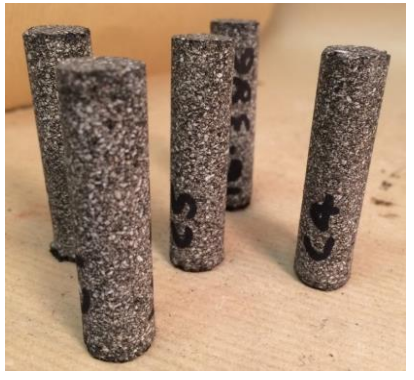

(b)

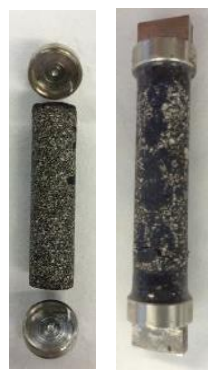

(c)

Figure 1. Procedure for the fabrication of testing specimens: (a) SGC specimen $150 \mathrm{~mm}$ in diameter, (b) FAM samples for DMA testing, and (c) specimens with grip holders ready for installation in the rheometer.

These specimens were used to apply the DMA testing technique which has been successfully used to characterise the viscoelastic properties (i.e. linear and non-linear), and the mechanical deterioration processes under cyclic loading (i.e. fatigue test) of FAM materials (Caro et al., 2012, 2008; Caro, Sánchez, \& Caicedo, 2014; Gudipudi \& Underwood, 2015; Masad et al., 2008, 2006, Tong, Luo, \& Lytton, 2013, 2015). The general procedure consists on using a rheometer with a special solid fixture configuration to conduct shear controlled strain tests on the small cylindrical FAM specimens. For the purposes of this study the following tests using the DMA configuration were conducted:

(1) frequency and temperature sweep tests to obtain the master curves of the materials, (2) sweep shear strain tests to determine the threshold that separates the non-linear viscoelastic zone to the zone where damage due to fatigue initiates and to identify the strain level that should be used to conduct fatigue tests, and (3) dynamic torsional tests 
under strain-controlled conditions, to characterise fatigue deterioration. The results from these tests were used to obtain some of the input parameters of the fracture model described in Section 4.

A Kinexus DSR rheometer by Malvern ${ }^{\circledR}$ was used to perform these tests. This geometry consists on an upper and lower geometry, a loading unit, and an environmental controller cartridge which slots into the rheometer holding the lower geometry on which the sample is placed, and a data acquisition system. Figure 2 illustrates the testing configuration in the rheometer.

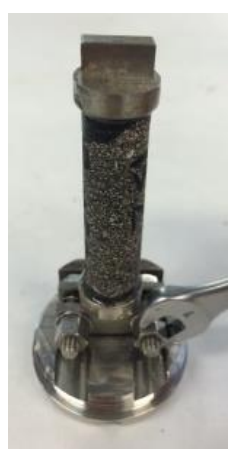

(a)

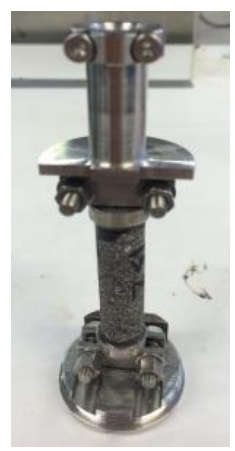

(b)

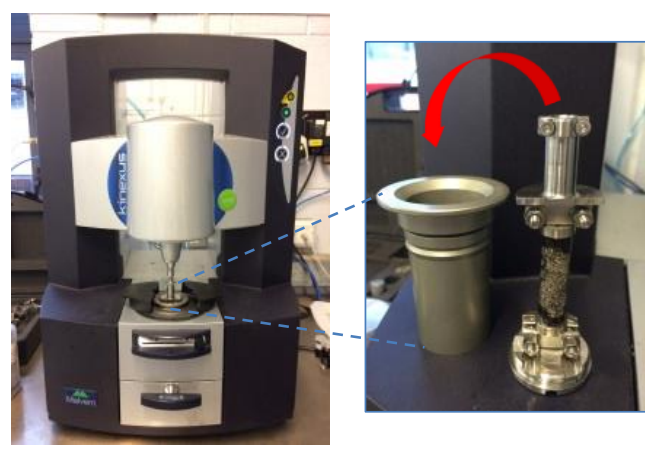

(c)

Figure 2. DMA testing configuration for FAM materials: (a) FAM sample with metallic holders fixed at the lower geometry, (b) FAM sample with metallic holders fixed at the upper and lower geometries and, (c) Mounting the FAM sample in the rheometer.

The master curves of the FAM-RAP materials are presented in the following section. The other two tests performed using this experimental configuration to obtain the parameters of the fracture model are presented in Section 5.

\subsection{Master curves of RAP-FAM mixtures}

Frequency and temperature sweep tests were conducted on the cylindrical FAM-RAP specimens to measure their complex modulus, $G^{*}$, for frequencies of $0.5-15.85 \mathrm{~Hz}$ at six temperatures $\left(15-65^{\circ} \mathrm{C}\right.$, with $10^{\circ} \mathrm{C}$ increments). These tests were conducted at a constant strain amplitude of $0.0065 \%$, a value commonly recommended in the literature to test FAM materials in their linear viscoelastic range (Caro et al., 2008, 2014; Castelo Branco, 
2008; Masad et al., 2008). Two replicates were tested for each mixture after observing low variability. Figure 3 presents the obtained average master curves of $G^{*}$, for all the FAM mixtures evaluated at a reference temperature of $25^{\circ} \mathrm{C}$.

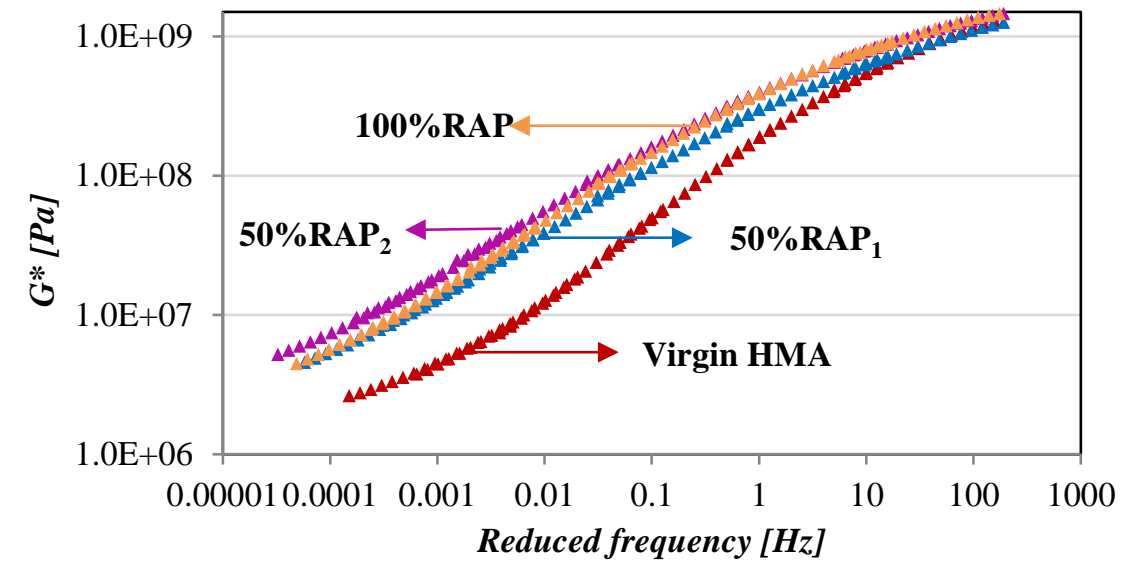

Figure 3. Master curves at a reference temperature of $25^{\circ} \mathrm{C}$ for the four FAM mixtures evaluated.

These figures reveal the effect of high-RAP contents (i.e. 50-100\%) on the $G^{*}$, of the FAM mixtures. All mixtures exhibited higher $\mathrm{G}^{*}$ values compared to the virgin HMA, as expected. For example, the incorporation of RAP material, in the $50 \% \mathrm{RAP}_{1}$ mixture manufactured with a soft virgin bitumen - increased $G^{*}$ by approximately $15.7 \%$, while the incorporation of the same RAP material in the mixture $50 \% \mathrm{RAP}_{2}$ - manufactured with the same virgin bitumen as the Virgin HMA - increased $G^{*}$ by $43.9 \%$, at a frequency of $10 \mathrm{~Hz}$ and a testing temperature of $25^{\circ} \mathrm{C}$. For the $100 \%$ RAP mixture, $G^{*}$ increased by $45.9 \%$ compared to the virgin HMA at the same frequency and testing temperature $(10 \mathrm{~Hz}$ and $25^{\circ} \mathrm{C}$ ). At lower loading frequencies (higher temperatures), these differences are even larger. These results are in agreement with previous results published by other researchers conducted on full asphalt mixtures (Li, Marasteanu, Christopher Williams, \& Clyne, 2008).

Furthermore, these results showed that the addition of the soft virgin bitumen in mixture $50 \% \mathrm{RAP}_{1}$ mitigated this hardening effect (exhibiting lower $\mathrm{G}^{*}$ values compared to those of the $50 \% \mathrm{RAP}_{2}$ ). However, the reduction in the stiffness provided by the soft 
bitumen $\left(50 \% \mathrm{RAP}_{1}\right)$ was expected to be more pronounced since, in theory, this mixture has similar properties to that of the virgin HMA. The results could be explained by the actual level of blending occurring between the old bitumen from RAP and the virgin bitumen, which is reflected in Figure 3. In this figure, the behaviour (i.e. $G^{*}$ values) of the virgin HMA and the $100 \%$ RAP materials are exhibited, and are significantly different. If complete blending occurs between the soft virgin bitumen and the bitumen from RAP (i.e. mixture $50 \% \mathrm{RAP}_{1}$ - having the same final penetration grade of the virgin HMA), the resultant mixture should have similar properties to that of the virgin HMA due to the contribution of the soft virgin bitumen. However, the $G^{*}$ values of this mixture are higher, ending up with a mixture with similar properties to that of the $100 \%$ RAP mixture, suggesting that complete blending did not occur between the old and new binders.

In addition, for the same RAP material, without changing the bitumen grade of the virgin bitumen (i.e. mixture 50\% $\mathrm{RAP}_{2}$ - containing equal amounts of the virgin HMA and $100 \%$ FAM-RAP materials present in Figure 3 ), the $G^{*}$ values were generally similar to those of the $100 \%$ RAP mixture. Therefore these results indicate that in both FAM mixtures containing 50\% RAP, regardless of the grade of the virgin bitumen, the behaviour of the final mixture was primary dominated by the hardened bitumen from RAP and little interaction with the virgin bitumen was obtained.

One hypothesis that may explain this behaviour is the internal structure of the FAM-RAP materials. Unlike conventional full asphalt mixtures, in which aggregates of different sizes coexist (i.e. coarse and fine particles) in these FAM materials, only fine particles (i.e. below $1 \mathrm{~mm}$ ) are present. Therefore, in full asphalt mixtures when RAP is incorporated and it behaves as a "black rock", where incomplete blending occurs, it acts as an intrusion of solid particles that influences the mix volumetrics, properties and 
performance through its aggregate gradation properties (McDaniel \& Anderson, 2001) Figure 4(a). However, within FAM materials, it is speculated that the FAM-RAP particles (having similar size) are bonded together with the bitumen forming clusters that influence the behaviour of the final mixture through their hardened bitumen properties. It is worth mentioning that the dominant effect of the hardened bitumen was observed over the entire range of loading frequencies and testing temperatures in Figure 3, being more pronounced at low frequencies. At certain conditions (high testing temperatures) these clumps could be fragmented. However, even if this happens, the properties of the mixtures are still dominated by the stiffer bitumen from RAP. Figure 4 shows a graphical representation of the intrusion of RAP material in full and FAM mixtures in the case where incomplete blending occurs.

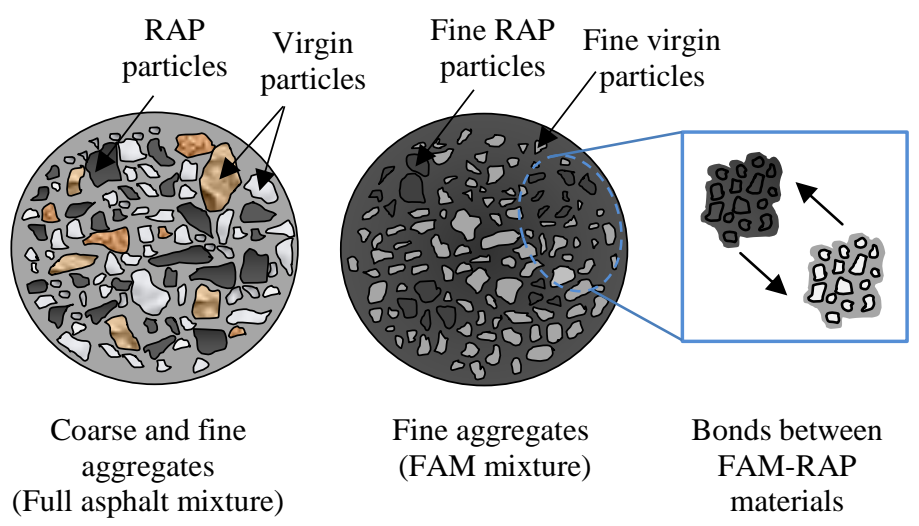

(a)

(b)

Figure 4. Influence of RAP on (a) full asphalt mixtures and, (b) FAM mixtures

\section{Analytical non-linear viscoelastic model for characterising fatigue damage}

The model used in this study was first developed by Lytton et al. (1993) for full HMA materials, later applied to evaluate the impact of moisture on these materials (Arambula, Masad, \& Martin, 2007), and then adapted for FAM materials (Masad et al., 2008). The model has also been successfully applied to evaluate moisture damage in these fine 
mixtures, as well as the fatigue performance of FAM warm mix asphalt (WMA) materials (Caro et al., 2012, 2008; Gudipudi \& Underwood, 2015; Tong et al., 2013, 2015). The model is based on the cumulative fatigue-related damage that the asphalt mixture experiences throughout its service life. In this way, it is possible to characterise the evolution of fatigue within the mixture during its service life (Lytton et al., 1993). The output parameter of the model is the crack growth index, denoted by $\Delta R$, which quantifies the average size of the crack length that is generated within the FAM specimens as a function of the number of load cycles $(N)$. Therefore, high values of $\Delta R$ through time are related to larger values of damage within the FAM samples.

The model is expressed in terms of the dissipated pseudo strain energy (DPSE), which refers to the energy that is exclusively spent in damage in viscoelastic materials. Particularly, it refers to the energy that is required to generate the crack to grow in the material. Therefore, the rate of change of the crack radius with the number of load applications is defined as (Arambula, Masad, \& Epps Martin, 2007) :

$$
\frac{d \bar{r}}{d N}=C\left(J_{R}\right)^{n}
$$

where $\bar{r}$ is the average crack radius in the sample, $N$ is the number of loading cycles, $C$, and $n$ are materials constants, and $J_{R}$ is the J-integral that quantifies the pseudo strain energy release rate per unit crack area, defined as:

$$
J_{R}=\frac{\frac{\partial W_{R}}{\partial N}}{\frac{\partial(c . s . a)}{\partial N}}
$$

where $W_{R}$ is the pseudo strain energy per unit volume of the initial mixture, and c.s.a. refers to the crack surface area. The ratio in the numerator represents the portion of the energy that is spent in damage for each loading cycle, and the ratio in the denominator is the change of the new surface area that is generated within the material by the 
appearance or propagation of the cracks in each cycle. $W_{R}$ is computed as the area in the hysteresis loop of the stress-pseudo strain region, which is typically assumed to be an ellipse. The relationship between $W_{R}$ and $N$ is typically of the form:

$$
W_{R}=a+b \operatorname{In}(N)
$$

where $a$ and $b$ are correlation factors. Equations (3) to (5) could be used to define the crack growth index parameter $(\Delta R(N))$; for details on the mathematical derivations used in this process, readers are referred to (Masad et al., 2008):

$$
\Delta R(N)=\left[(2 n+1)^{n+1}\left(\frac{G_{R} b}{4 \pi G_{1} \Delta G_{f}}\right)^{n} N\right]^{\frac{1}{2 n+1}}
$$

where $n$ is related to the relaxation time, $G_{l}$ is the initial relaxation modulus, $G_{R}$ represents a reference modulus, which corresponds to the threshold at which the material transits from the non-linear viscoelastic behaviour to the damage zone, $b$ represents the rate of change of the pseudo strain energy as a function of the loading cycles $(N)$, which is calculated from equation (5), and $\Delta G_{f}$ represents the work of adhesion between the bitumen and the aggregates, which is computed from the individual Surface Free Energy (SFE) properties of the mixture components (i.e., virgin bitumen, old bitumen from RAP, blended virgin and RAP bitumen, and aggregates). Section 5 presents more details about these parameters.

The main advantage of this model is that unlike the conventional fatigue tests where only information about the final damage state of the material is provided (e.g. number of loading cycles to failure), in this case the history of the fatigue degradation within the material before failure is captured. This, in turn, provides useful information to understand the mechanical degradation mechanisms caused by cyclic loading within the mixture in the field; information that might also be useful to support the design of 
maintenance and rehabilitation programs. The next section presents the experimental procedures performed to obtain the required input parameters of this model.

\section{Experimental testing procedures to characterise fatigue degradation}

The following sections describe the tests that were conducted to obtain the required parameters to compute the crack growth index $\Delta R(N)$ for the FAM and FAM-RAP mixtures.

\subsection{Tests conducted using the DMA configuration on the FAM-RAP mixtures}

The following are the test procedures conducted on the FAM and RAP-FAM specimens using the DMA configuration previously described to compute the crack growth index $\Delta R(N)$ in equation 6.

- Frequency and temperature sweep tests: this test procedure has already been described in Section 3. The results from this test were initially used to construct the master curves of the mixtures (Section 3) and then, to predict the viscoelastic material behaviour in the time domain from these curves in order to obtain the relaxation modulus of the materials. With this information, the initial relaxation modulus, $G_{l}$ and the parameter $n$ in Equation 6 were determined. This procedure was performed by using a Prony series (Park \& Schapery, 1999; Schapery, 1961). After the relaxation curves for each FAM mixture were obtained, they were fitted to the power law equation given by (Kim, 2009):

$$
G(t)=G_{0}+G_{1} t^{-m}
$$

where, the initial relaxation modulus, $G_{1}$ - included in Equation 6 - and the slope of the relaxation curve $m$ are fitting constants. The parameter $n$ in Equation 7 was determined from $m$ by applying the relationship $n=1+1 / m$ (Masad et al., 2008). 
- Shear strain sweeps: strain sweep tests were conducted at $28^{\circ} \mathrm{C}$, using a constant frequency of $10 \mathrm{~Hz}$ and six different strain levels $(0.001 \%, 0.005 \%, 0.01 \%, 0.05 \%$, $0.1 \%$ and $0.15 \%$ strain) with 2 minutes duration at each strain level. Two replicates were tested for each mixture. This test protocol has been successfully used in previous studies (Caro et al., 2014; Castelo Branco, 2008) for FAM materials. Information from this test was used to: (1) identify thresholds at which the material transits from a non-linear response to a damage zone - which provides the $G_{R}$ value included in Equation 6 - that is specific for each mixture, and (2) to define a threshold strain level that was able to generate damage in the material for further fatigue characterisation. Figure 5 presents a typical result of this test, where the transition from the non-linear viscoelastic behaviour (i.e. $G^{*}$ values remaining almost constant during the 2-min tests at the tested strain level - left part of the graph) to damage (i.e. when the modulus starts decreasing during the 2-min test at a specific shear strain value - right part of the graph) can be easily identified.

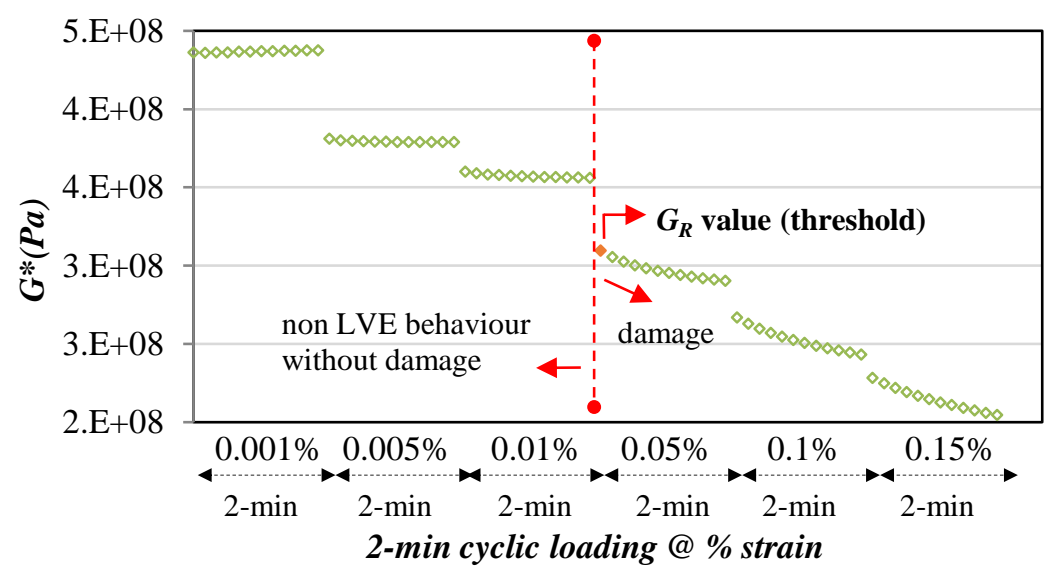

Figure 5. Typical result of shear strain sweep tests at $28^{\circ} \mathrm{C}$

- Shear oscillation or fatigue: this test has been successfully used in previous studies (Caro et al., 2012, 2008, 2014, Masad et al., 2008, 2006; Montepara et al., 2011) and it consists of applying a cyclic loading condition under a constant shear 
strain amplitude - determined from the previous strain sweep test - for long periods of time. During the test, the specimen deteriorates with the progression of the number of load cycles, $N$, which is reflected by a decrease in the value of $G^{*}$ and an increase in the value of $\delta$. The tests were conducted at $28^{\circ} \mathrm{C}$ and $10 \mathrm{~Hz}$, applying a constant shear strain value of $0.09 \%$, for $4 \mathrm{~h}$. Five replicates were tested for each mixture. The results from the test are used to quantify the parameter $b$ in Equation 6, by using Equation 5. In addition, the number of cycles to failure $N_{f}$ for each mixture is also commonly determined from this part of the test. Although this quantity is not an input parameter of the $\Delta R$ model, it is still important to compare the final degradation of the different mixtures at the end of the dynamic shear test. Generally, this parameter is taken as the number of cycles at which failure occurs within the sample, which is identified by a sudden simultaneous drop of $G^{*}$ and $\delta$ (Castelo Branco, 2008). However, within the 4 hours tests, the RAP materials did not exhibit full failure, so the parameter $N_{f}$ was replaced by the number of cycles at which $G^{*}$ decreased a certain amount. After analysing the data, it was observed that in all the samples, $G^{*}$ decreased at least $40 \%$ with respect to its initial value. Therefore, in this study, the number of cycles at which

$G^{*}$ decreased $40 \%$ of its initial value $\left(N_{40}\right)$ was selected as the parameter to compare the final damage generated within the FAM mixtures at the end of the cyclic loading test.

\subsection{Tests conducted to estimate the adhesive bond within the RAP-FAM mixtures}

The $S F E$ of a material is defined as the amount of energy required to create a new unit of surface area of that material in vacuum conditions (Bhasin, Little, Vasconcelos, \& Masad, 
2007). According to the Good-Van Oss-Chaudhury theory (Van Oss, Chaudhury, \& Good, 1988), the $S F E$ of a material comprises a non-polar component $\gamma^{L W}$ (Lifshitz-van der Waals) and a polar component $\gamma^{A B}$, which is composed of a monopolar acidic component $\gamma^{+}$, and a monopolar basic component $\gamma$ as follows:

$$
S F E=\gamma^{L W}+\gamma^{A B}=\gamma^{L W}+2 \sqrt{\gamma^{+} \gamma^{-}}
$$

The $S F E$ properties of the individual components (i.e. bitumen and aggregates) present in all the FAM mixtures can be used to determine the amount of energy required to separate the bonds that hold these materials together to create a new unit of area of each material - which corresponds to the parameter $\Delta G_{f}$ in Equation 6 - through the following equation:

$$
\Delta G_{f}=2 \sqrt{\gamma_{A}^{L W} \gamma_{S}^{L W}}+2 \sqrt{\gamma_{A}^{+} \gamma_{S}^{-}}+2 \sqrt{\gamma_{A}^{-} \gamma_{S}^{+}}
$$

where the subscripts $A$ and $S$ stand for the bitumen and the aggregates, respectively.

Thus in this study, the $S F E$ properties of the specific bitumen present in each mixture and the virgin limestone aggregates (the same for all the mixtures) were measured in order to include all the adhesion cases from all the evaluated mixtures. These adhesion cases include: 1) virgin bitumen and the virgin aggregates (i.e. for the virgin HMA mixture), 2) recovered bitumen from RAP and the virgin aggregates (i.e. for the $100 \%$ RAP mixture) and, 3) the final bitumen blend resulting from the combination of the recovered bitumen from RAP with the virgin bitumen, and the aggregates (i.e. for the two mixtures containing 50\% RAP).

A Dynamic Contact Angle (DCA) analyser and a Dynamic Vapour Sorption (DVS) system were used to determine the $S F E$ components of the bitumen present in each FAM mixture and the $S F E$ of the limestone aggregates, respectively. Details about these 
tests can be found elsewhere (Bhasin 2006; Bhasin et al. 2006; Ahmad \& Airey 2010a; Ahmad \& Airey 2010b)

\section{Experimental results}

\subsection{Relaxation modulus and damage properties of the FAM-RAP mixtures}

The mean values and the standard deviation $(\sigma)$ of the time-domain viscoelastic properties and fatigue degradation properties of the FAM-RAP mixtures obtained from the DMA tests are presented in Table 5. The table also includes the average number of cycles to fatigue degradation $N_{40}$ (i.e. number of cycles to reach $40 \%$ of decay of the initial $G^{*}$ ) for each mixture.

Table 5. Average viscoelastic and damage properties of the FAM-RAP mixtures

\begin{tabular}{|c|c|c|c|c|c|c|c|c|c|}
\hline \multirow{2}{*}{ Test } & \multirow{2}{*}{ Parameter } & \multicolumn{2}{|c|}{ Virgin HMA } & \multicolumn{2}{|c|}{$50 \% \mathrm{RAP}_{1}$} & \multicolumn{2}{|c|}{$50 \% \mathrm{RAP}_{2}$} & \multicolumn{2}{|c|}{$100 \%$ RAP } \\
\hline & & Mean & $\sigma$ & Mean & $\sigma$ & Mean & $\sigma$ & Mean & $\sigma$ \\
\hline $\begin{array}{l}\text { Frequency } \\
\text { and T }\end{array}$ & $G_{*}\left(10^{7} \mathrm{~Pa}\right)$ & 1.74 & - & 7.27 & - & 9.88 & - & 8.74 & - \\
\hline sweep & $m^{*}$ & 0.32 & - & 0.42 & - & 0.43 & - & 0.45 & - \\
\hline tests & $n^{*}$ & 4.13 & - & 3.38 & - & 3.33 & - & 3.22 & - \\
\hline $\begin{array}{l}\text { Dynamic } \\
\text { amplitude } \\
\text { sweep test }\end{array}$ & $G_{R}\left(10^{8} \mathrm{~Pa}\right)$ & 3.08 & ${ }_{2}^{1.75 \times 10^{-}}$ & 3.84 & ${ }_{2}^{2.12 \times 10^{-}}$ & 4.76 & $1.4 \times 10^{-2}$ & 5.11 & $\begin{array}{l}7.78 \times 10 \\
2\end{array}$ \\
\hline $\begin{array}{l}\text { Shear } \\
\text { oscillation } \\
\text { test }\end{array}$ & $\begin{array}{l}b \\
\mathrm{~N}_{40}\left(10^{4}\right.\end{array}$ & 22.07 & 1.64 & 2.26 & 3.65 & 49.56 & 8.24 & 46.81 & 4.19 \\
\hline & cycles) & 5.91 & 3.16 & 7.16 & 4.17 & 2.45 & $3.5 \times 10^{-4}$ & 2.57 & 0.10 \\
\hline
\end{tabular}

*values obtained from the average master curves of each mixture.

\subsection{SFE results}

Table 6 presents the $S F E$ results of each bitumen present in the FAM mixtures as well as those of the limestone aggregates. These values, were used to calculate the work of adhesion - parameter $\Delta G_{f}$ in Equation 6 - by means of Equation 9, for each corresponding bitumen-aggregate interfaces in all the FAM mixtures.

Table 6. SFE components for the constituent materials of the mixtures (values in $\mathrm{mJ} / \mathrm{m}^{2}$ )

\begin{tabular}{lcccccc}
\multicolumn{1}{c}{ Material } & $\gamma^{+}$ & $\gamma$ & $\gamma^{L W}$ & $\gamma^{A B}$ & $S F E$ & $\Delta G_{f}$ \\
\hline Virgin bitumen & 0.28 & 1.52 & 18.89 & 1.30 & 20.18 & 74.16 \\
Recovered bitumen & 0.10 & 6.47 & 13.47 & 1.61 & 15.07 & 64.61
\end{tabular}




\begin{tabular}{|c|c|c|c|c|c|c|}
\hline Bitumen blend from $\mathrm{RAP}_{1}$ & 0.02 & 1.91 & 29.28 & 0.39 & 29.62 & 90.82 \\
\hline Bitumen blend from $\mathrm{RAP}_{2}$ & 0.02 & 1.85 & 23.95 & 0.41 & 24.36 & 82.37 \\
\hline Limestone aggregates & 0.59 & 1.50 & 66.65 & 1.88 & 68.54 & - \\
\hline
\end{tabular}

\section{7. $\Delta R$ model results and analysis}

Based on the parameters presented in Table 5 and Table 6, and using Equation 6, the average crack growth index, $\Delta R(N)$, for the FAM and RAP-FAM mixtures was computed and plotted as a function of the number of loading cycles (Figure 6). The plots of $\Delta R(N)$ were constructed up to the 4 hours test duration. The dots in this figure represent the parameter $N_{40}$ (defined later in this section).

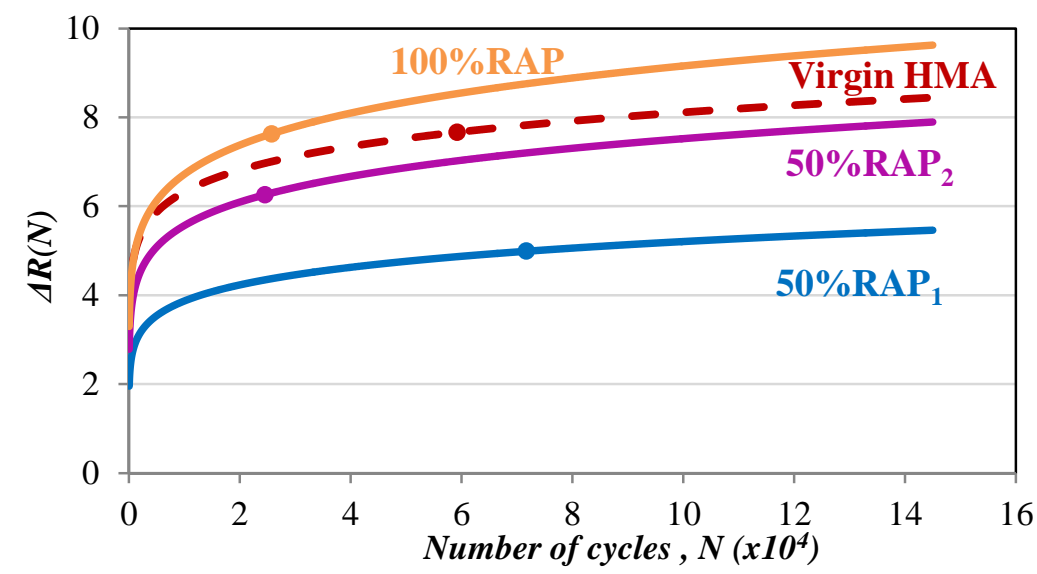

Figure 6. Average results of $\Delta R(N)$ as a function of $N$ in the FAM-RAP mixture

These results suggest that in terms of the $\Delta R(N)$, the material exhibiting the best mechanical performance to fatigue degradation (i.e. lowest values of $\Delta R$ ) is the $50 \% \mathrm{RAP}_{1}$, followed by the $50 \% \mathrm{RAP}_{2}$, the virgin HMA and the $100 \%$ RAP mixtures. The virgin HMA was expected to present the best mechanical performance, however these results indicate that the addition of 50\% RAP resulted in mixtures with lower cumulative fracture damage. However, further increments in the RAP content (100\% RAP mixture), where no virgin materials are present, resulted in mixtures with high cumulative fracture damage (i.e. highest values of $\Delta R$ ), as expected. 
Furthermore, in terms of the 50\% RAP mixtures, the addition of a softer virgin bitumen $\left(50 \% \mathrm{RAP}_{1}\right)$ improved the mechanical performance of the mixture, as depicted by the smallest $\Delta R(N)$ values in Figure 6. Recalling from the results of the viscoelastic properties of the FAM mixtures, it was observed that the performance of the mixtures containing 50\% RAP was dominated by the hardened bitumen from RAP, and complete blending did not occur. Thus, this behaviour indicates that if blending occurs at a certain extent, then a softer bitumen contributes to mitigate the fatigue resistance of FAM mixtures containing 50\% RAP. Furthermore, the addition of 50\% RAP material with no change in the penetration grade of the virgin bitumen $\left(50 \% \mathrm{RAP}_{2}\right)$, also improved the fatigue resistance of the FAM mixture, although to a lesser extent. These results are important since they suggest that high amounts of RAP material (i.e. up to 50\%) might be used as part of regular HMA mixtures without compromising the resistance of its fine matrix to deterioration under cyclic loading. However, it is worth mentioning that these results are only valid for the selected strain level.

Additionally, this information is useful to evaluate the performance of each mixture individually. For instance, a comparison of $N_{40}$ (i.e. number of cycles to decrease the $G^{*}$ to $40 \%$ of its initial value), as shown in Figure 7 , suggests that the $100 \%$ RAP and $50 \% \mathrm{RAP}_{2}$ mixtures reached $40 \%$ of initial $G^{*}$ at the lowest number of cycles $\left(2.57 \times 10^{4}\right.$ and $2.45 \times 10^{4}$, respectively), while the virgin $\mathrm{HMA}$ and $50 \% \mathrm{RAP}_{1}$ mixture presented the same percentage of initial $G^{*}$ at a higher number of loading cycles $\left(5.91 \times 10^{4}\right.$ and $7.16 \times 10^{4}$, respectively). 


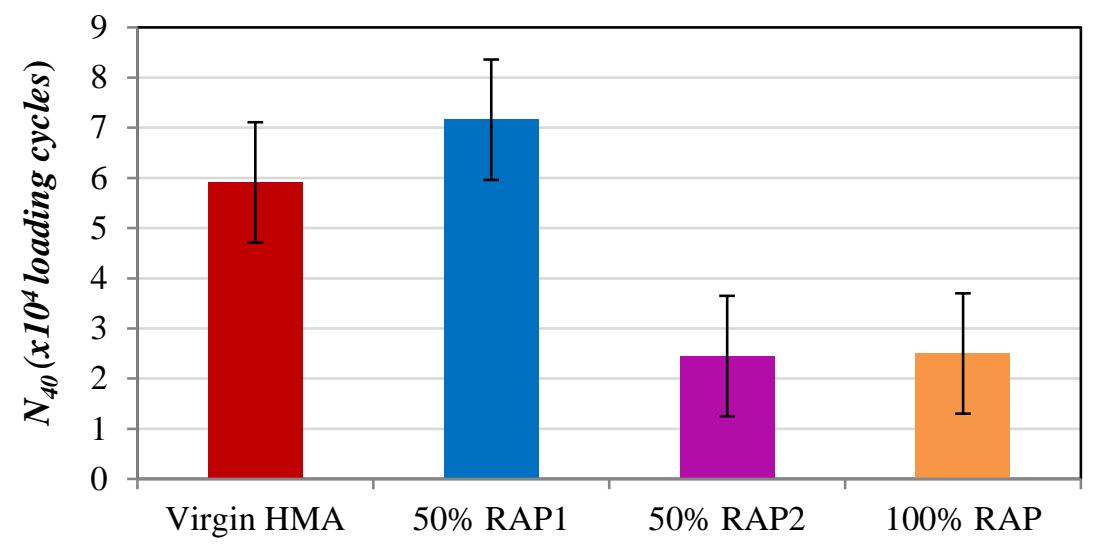

Figure 7. Comparison of $N_{40}$ for all the FAM mixtures

This information, along with the $\Delta R(N)$ curves in Figure 6 , is useful to evaluate the overall response of each mixture under cyclic loading in terms of the relative damage generated in their internal structure and their fatigue resistance. In terms of $N_{40}$, the material exhibiting the highest $N_{40}$ value was the $50 \% \mathrm{RAP}_{1}$, followed by the virgin HMA, the $100 \% \mathrm{RAP}$, and the $50 \% \mathrm{RAP}_{2}$. These results are not in good agreement with those obtained from the parameter $\Delta R(N)$, except for the $50 \% \mathrm{RAP}_{1}$. This suggests that all the FAM mixtures evaluated are affected in a different way by the RAP material, when evaluating the amount of accumulated damage generated in their internal structure and the final resistance condition of the material. In other words, these results suggest that the number of cycles required to achieve a certain reduction in the modulus of the material is not enough to fully characterise fatigue processes in these materials, since the degradation mechanisms occurring in the mixtures that produce the loss in the resistance of the mixtures are equally relevant.

For instance, the virgin HMA presented high values of $\Delta R(N)$ (high internal damage) as shown in Figure 6, and a high $N_{40}$ value (Figure 7). Moreover, the $100 \%$ RAP and $50 \% \mathrm{RAP}_{2}$ mixtures presented similar $N_{40}$ values, however their mechanical performance $\left(\Delta R(N)\right.$ values) were different. Nonetheless, the $50 \% \mathrm{RAP}_{1}$ mixture showed good correlation with the results obtained from the parameter $\Delta R(N)$, as it presented the 
least internal damage (lowest values of $\Delta R(N)$ ) and the highest $N_{40}$ value. If the tendency of the $N_{40}$ values is still maintained over the loading of the mixtures, they are expected to correlate with the actual $N_{f}$ values (i.e. number of cycles to failure or fatigue life of the material), which means that the materials exhibiting lower values of $N_{40}$ will fail before those exhibiting larger values of $N_{40}$.

The desired condition of the performance of a mixture in field is that where the material experiences less or small amounts of damage in its internal structure (low $\Delta R(N)$ values), and it also presents an extended fatigue resistance (high $N_{40}$ value). In this way, the durability of the material will be enhanced. Therefore, if this information is known, it will contribute to a better understanding of the performance of the materials, thus promoting their responsible use in road infrastructure projects.

In this study the incorporation of 50\% RAP in FAM materials was not found to affect the resistance of the mixture to fatigue damage at the selected strain level. In particular, as mentioned before, the use of $50 \%$ RAP in combination with a soft bitumen $\left(50 \% \mathrm{RAP}_{1}\right)$ resulted in better fatigue performance compared to that of the mixture manufactured with the same RAP material fabricated without the soft virgin bitumen $\left(50 \% \mathrm{RAP}_{2}\right)$. Also, this mixture was the only one that exhibited good agreement with the parameter $\Delta R(N)$, as it showed the highest $N_{40}$ value as well. This condition might represent an advantage for the improvement of the road however, these results are only valid for the selected strain and it is worth mentioning that at other strain levels this tendency might be inverted or changed. At last, replacing the virgin HMA by $100 \%$ RAP material was shown to negatively affect the fatigue resistance of the material, since even though the $N_{40}$ value is low compared to that of the virgin HMA, the overall fatigue resistance of the mixture is worsened. 


\section{Conclusions and recommendations}

This study evaluated three aspects to quantify the effect of high-RAP content on the rheological and fatigue deterioration properties of two FAM mixtures containing 50\% RAP material in combination with virgin materials (with bitumen at two different penetration grade binders), one FAM mixture containing 100\% RAP mixture and an unmodified control FAM-HMA, which was included for comparison. The first aspect included the viscoelastic properties of the mixtures by means of master curves. The second aspect included the fatigue degradation properties by means of a fracture model whose final output parameter was the crack growth index, $\Delta R(N)$ that quantifies the cumulative damage in the internal structure of the material when subjected to cyclic loading. Finally, the third aspect included the change in the fatigue resistance, which was defined as the number of cycles to decrease to $40 \%$ of the initial complex modulus of the mixture. All these aspects were evaluated by means of DMA testing conducted on cylindrical specimens representing the FAM existing within full mixtures. SFE tests were also conducted on the constitutive materials of each mixture in order to obtain one of the input parameters for the $\Delta R(N)$ that accounts for the quality of the adhesion between the components of the mixtures.

Results from the first aspect showed that, in general, the incorporation of highRAP contents (50 and 100\% RAP) resulted in stiffer mixtures, as expected. The use of a soft bitumen in combination with 50\% RAP material mitigated this effect, since the difference between the $G^{*}$ of the $50 \% \mathrm{RAP}_{1}, 50 \% \mathrm{RAP}_{2}$ and $100 \% \mathrm{RAP}$ mixtures with the virgin $\mathrm{HMA}$ were $15.7,43.9$ and $45.9 \%$ respectively at $10 \mathrm{~Hz}$ and $25^{\circ} \mathrm{C}$. However, the contribution of the soft bitumen was expected to reduce the stiffening effect to a higher amount by producing a mixture with similar properties to that of the virgin HMA. Nonetheless, the RAP material dominated the final behaviour in all the FAM-RAP 
mixtures. This dominant effect suggests that complete blending between the old bitumen from the RAP and the virgin bitumen did not occur. Also, it is hypothesised that within FAM materials, the particles are bonded together with the bitumen forming clusters that influence the behaviour of the final mixture through the hardened bitumen properties explaining the dominant effect of the RAP bitumen. This behaviour was observed over all the ranges of loading frequencies and testing temperatures.

Results from the crack growth index model, showed that the mixtures containing $50 \%$ RAP exhibited a smaller cumulative damage compared to the virgin HMA. On the contrary, the $100 \%$ RAP mixture exhibited the highest cumulative damage from all the FAM mixtures. Furthermore, a comparison of $N_{40}$, showed that the $100 \%$ RAP and $50 \%$ $\mathrm{RAP}_{2}$ mixtures reached $40 \%$ of the initial $G^{*}$ at the lowest number of cycles, while the virgin $\mathrm{HMA}$ and $50 \% \mathrm{RAP}_{1}$ mixture presented the same percentage reduction in $G^{*}$ at a higher number of loading cycles. These results showed that there was no agreement between these two parameters, except for the $50 \% \mathrm{RAP}_{1}$ mixture, suggesting that the incorporation of high-RAP contents produced a different effect in the amount of damage generated in the internal structure and the final fatigue resistance of the material for each of the RAP-FAM mixtures.

In summary, the overall results from this study suggest that although the incorporation of 50\% RAP content increased the stiffness of the RAP-FAM mixtures, this hardening effect did not necessarily result in mixtures with inferior fatigue performance in the FAM mixtures containing 50\%RAP at the selected strain level. However, higher amounts of RAP content (i.e. 100\%) resulted in mixtures with less fatigue resistance compared to the 50\% RAP and virgin HMA mixtures. Also, the use of a soft virgin bitumen mitigated the stiffening effect provided by the hardened RAP bitumen and 
improved the fatigue performance of the FAM mixtures, even if complete blending between the old-new binders did not occur.

It was also observed that the FAM mixtures were affected in a different way by the RAP material, in terms of the relatively damage generated in their internal structure (parameter $\Delta R(N)$ ) as well as in their fatigue resistance (parameter $N_{40}$ ). These results suggest that both parameters are equally important to evaluate the material performance as they provide different, but complementary information, which contributes to support maintenance decisions to enhance the durability of these materials in field.

It is worth mentioning that in this study the properties of the RAP material were controlled, which represents the ideal material to be reclaimed in asphalt mixtures. However, in practice the properties of the RAP material might be unknown and the variability of its properties restrains the possibility of providing generalised conclusions on the overall rheological and mechanical performance of mixtures containing RAP material. Also, it is noteworthy that the comparative results obtained in terms of the fatigue degradation of the FAM-RAP mixtures are only valid for the selected strain level, and they may vary at other strain values.

\section{Acknowledgments}

The first author would like to acknowledge Colciencias for the funding provided through the program No. 617-2013 for doctoral studies abroad.

\section{References}

Ahmad, N., \& Airey, G. D. (2010a). Dynamic Contact Angle Measurements of Bitumen using a Wilhelmy Plate Device. NTEC Report Number: 11086 (Vol. 364). Nottingham, UK.

Ahmad, N., \& Airey, G. D. (2010b). Dynamic Vapour Sorption Measurement Techniques for Aggregates. NTEC Report Number: 11087 (Vol. 364). Nottingham, UK.

Anderson, E. D., \& Daniel, J. S. (2013). Long Term Performance of High RAP Pavements: Case Studies. Transportation Research Record: Journal of the Transportation Research Board. 
Arambula, E., Masad, E., \& Epps Martin, A. (2007). Moisture susceptibility of asphalt mixtures with known field performance using dynamic analysis and a crack growth model. Journal of the Transportation Research Board, No. 2001, 20-28.

Arambula, E., Masad, E., \& Martin, A. E. (2007). Influence of Air Void Distribution on the Moisture Susceptibility of Asphalt Mixes. Journal of Materials in Civil Engineering, 655664.

Bell, C. A. (1989). Summary report on aging of asphalt-aggregate systems. Corvallis, OR.

Bennert, T., Daniel, J. S., \& Mogawer, W. (2014). Strategies for incorporating higher RAP percentages: Review of Northeast States Implementation Trials. Transportation Research Record: Journal of the Transportation Research Board, 1-19.

Bhasin, A. (2006). Development of methods to quantify bitumen-aggregate adhesion and loss of adhesion due to water. Texas A\&M University.

Bhasin, A., Little, D. N., Vasconcelos, K. L., \& Masad, E. (2007). Use of Surface Free Energy to Identify Moisture Sensitivity of Materials for Asphalt Mixes. Transportation Research Record, (2001), 37-45.

Bhasin, A., Masad, E., Little, D., \& Lytton, R. (2006). Limits on adhesive bond energy for improved resistance of hot-mix asphalt to moisture damage. Transportation Research Record: Journal of the Transportation Research Board (1970), 3-13.

Bonaquist, R. (2005). Laboratory evaluation of hot mix asphalt (HMA) mixtures containing recycled or waste product materials using performance testing.

BS 2000-49:2007. Bitumen and bituminous binders - Determination of needle penetration.

BS 2000-505:2010. Bitumen and bituminous binders - Determination of dynamic viscosity of bituminous binder using a rotating spindle apparatus.

BS 2000-58:2007. Bitumen and bituminous binders — Determination of the softening point Ring and Ball method.

BS 2000-80:2007. Bitumen and bituminous binders — Determination of the Fraass breaking point.

BS 4987-1:2005. Coated macadam (asphalt concrete) for roads and other paved areas -Part 1: Specification for constituent materials and for mixtures.

BS EN 12697-31:2007. Bituminous Mixtures-Test Methods for hot mix asphalt- Part 31: Specimen preparation by gyratory compactor.

BS EN 12697-4:2005. Bituminous mixtures-Test methods for hot mix asphalt-Part 4: Bitumen recovery: Fractionating column.

Caro, S., Beltrán, D. P., Alvarez, A. E., \& Estakhri, C. (2012). Analysis of moisture damage susceptibility of warm mix asphalt (WMA) mixtures based on Dynamic Mechanical Analyzer (DMA) testing and a fracture mechanics model. Construction and Building Materials, 460-467.

Caro, S., Masad, E., Airey, G., Bhasin, A., \& Little, D. (2008). Probabilistic Analysis of Fracture in Asphalt Mixtures Caused by Moisture Damage. Transportation Research Record: Journal of the Transportation Research Board, 28-36.

Caro, S., Sánchez, D. B., \& Caicedo, B. (2014). Methodology to characterise non-standard asphalt materials using DMA testing: application to natural asphalt mixtures. International Journal of Pavement Engineering, 1-10.

Castelo Branco, V. T. F. (2008). A unified method for the analysis of nonlinear viscoelasticity and fatigue cracking of asphalt mixtures using the dynamic mechancial analyzer. Texas 
A\&M Univeristy.

Copeland, A. (2011). Reclaimed asphalt pavement in asphalt mixtures: State of the practice. Publication No. FHWA-HRT-11-021.

Daniel, J., Pochily, J., \& Boisvert, D. (2010). Can more reclaimed asphalt pavements be added? Study of extracted binder properties from plant produced mixtures with up to $25 \%$ reclaimed asphalt pavement. Transportation Research Record: Journal of the Transportation Research Board, No 2180.

Gudipudi, P., \& Underwood, B. S. (2015). Testing and modeling of fine aggregate matrix and its relationship to asphalt concrete mix. Transportation Research Record: Journal of the Transportation Research Board.

Hajj, E. Y., Sebaaly, P. E., \& Shrestha, R. (2007). A laboratory evaluation on the use of recycled asphalt pavements in HMA mixtures. Reno, Nevada.

He, Y., Alavi, M. Z., Jones, D., \& Harvey, J. (2016). Proposing a solvent-free approach to evaluate the properties of blended binders in asphalt mixes containing high quantities of reclaimed asphalt pavement and recycled asphalt shingles. Construction and Building Materials, 114, 172-180.

Huang, B., Li, G., Vukosavljevic, D., Shu, X., \& Egan, B. (2005). Laboratory Investigation of Mixing Hot-Mix Asphalt with Recycled Asphalt Pavement. Transportation Research Record: Journal of the Transportation Research Board, 1-19.

Kim, R. J. (2009). Modeling of asphalt concrete (1st ed.). New York: MacGraw-Hill.

Li, X., Marasteanu, M., Christopher Williams, R., \& Clyne, T. R. (2008). Effect of RAP (proportion and type ) and binder grade on the properties of asphalt mixtures. Transportation Research Record: Journal of the Transportation Research Board, 90-97.

Lytton, R. L., Uzan, J., Fernando, E. G., Roque, R., Hiltunen, D., \& Stoffels, S. M. (1993). Development and validation of performance prediction models and specifications for asphalt binders and paving mixes. SHRP-A-357. Washington, DC.

Masad, E., Castelo Branco, V. T. F., Little, D. N., \& Lytton, R. (2008). A unified method for the analysis of controlled-strain and controlled-stress fatigue testing. International Journal of Pavement Engineering, 233-246.

Masad, E., Zollinger, C., Bulut, R., Little, D., \& Lytton, R. (2006). Characterization of HMA moisture damage using surface energy and fracture properties. Journal of the Association of Asphalt Paving Technologists (AAPT), 713-754.

Maupin, G. W., Diefenderfer, S., \& Gillespie, J. (2008). Evaluation of using higher percentages of recycled asphalt pavement in asphalt mixes in Virginia. Richmond, VA.

McDaniel, R., \& Anderson, R. M. (2001). NCHRP Report 452. Recommended use of reclaimed asphalt pavement in the superpave mix design method: technician's manual. Washington, DC.

McDaniel, R., Shah, A., Huber, G., \& Gallivan, V. (2007). Investigation of properties of plantproduced RAP mixtures. Transportation Research Record: Journal of the Transportation Research Board, No. 1998.

Mogawer, W., Bennert, T., Daniel, J. S., Bonaquist, R., Austerman, A., \& Booshehrian, A. (2012). Performance characteristics of plant produced high RAP mixtures. Road Materials and Pavement Design.

Montepara, A., Romeo, E., Isola, M., \& Tebaldi, G. (2011). The role of fillers on cracking behavior of mastics and asphalt mixtures. Journal of the Association of Asphalt Paving Technologists (AAPT), 161-90. 
Oke, O. O. (2011). A study on the development of guidelines for the production of bitumen emulsion stabilised RAPs for roads in the tropics. The University of Nottingham.

Park, S. W., \& Schapery, R. A. (1999). Methods of interconversion between linear viscoelastic material functions. Part I: A numerical method based on Prony series. International Journal of Solids Structures.

Schapery, R. A. (1961). A simple collocation method for fitting viscoelastic models to experimental data. Rep. GALCIT SM 61-23A. Pasadena, CA: California Institute of Technology.

Tabakovi, A., Gibney, A., Mcnally, C., \& Gilchrist, M. D. (2010). Influence of Recycled Asphalt Pavement on fatigue performance of asphalt concrete base courses, 643-650.

Tong, Y., Luo, R., \& Lytton, R. L. (2013). Modeling water vapor diffusion in pavement and its influence on fatigue crack growth of fine aggregate mixture. Transportation Research Record: Journal of the Transportation Research Board.

Tong, Y., Luo, R., \& Lytton, R. L. (2015). Moisture and aging damage evaluation of asphalt mixtures using the repeated direct tensional test method. International Journal of Pavement Engineering.

Van Oss, C., Chaudhury, M., \& Good, R. (1988). Interfacial Lifshitz-Van der Waals and polar interactions in macroscopic systems. 